\title{
VIABILIDADE ECONÔMICA DA IMPLANTACCÃO DE SOJA E FEIJÃO COM SUCESSÃO DE MILHO NO SUDESTE DE GOIÁS
}

Iarla Bruna Oliveira de Almeida ${ }^{1}$, Daniela Gonçalves Rosa ${ }^{1}$, Rafhael Zander Lopes Ferreira$^{1}$, Andrécia Cósmem da Silva $^{2}$, Matheus da Silva Araújo ${ }^{3}$

${ }^{1}$ Discentes do Curso de Agronomia da Universidade Estadual de Goiás, Campus Ipameri, Ipameri, GO, Brasil. (iarlaalmeida96@hotmail.com)

${ }^{2}$ Professora mestre da Universidade Estadual de Goiás, Campus Ipameri, Ipameri, GO, Brasil

${ }^{3}$ Mestrando do Curso de Pós-Graduação em Ciências Florestais da Universidade de Brasília, Campus Darcy Ribeiro, Brasília, DF, Brasil

Recebido em: 08/04/2017 - Aprovado em: 10/06/2017 - Publicado em: 20/06/2017 DOI: 10.18677/EnciBio_2017A103

\begin{abstract}
RESUMO
O presente trabalho teve como objetivo analisar a viabilidade econômica da implantação do cultivo da soja e do feijão com sucessão do milho, no Município de Orizona, Goiás. A propriedade possui área total de 203,08 ha. Para análise econômica foram utilizados 130 ha, sendo 80 ha para o cultivo da soja e 50 ha para o cultivo do feijão na safra e após a colheita foi cultivado safrinha com a cultura do milho nas duas áreas. Para o estudo de viabilidade utilizou os seguintes indicadores econômicos: Índice Benefício Custo (IBC), Valor Presente Líquido (VPL) e aplicação do Payback econômico do capital investido. A taxa de retorno utilizada foi de $10 \% \mathrm{e}$ o tempo de diagnóstico de 5 anos. Obteve-se um custo total para a implantação do projeto de $R \$$ 3.133.188,59, enquanto que a receita atingiu $R \$ 5.025 .773,97$, obtendo lucro líquido de $R \$ 1.892,585,38$. O Payback foi verificado aos 6 meses e a razão IBC foi de 1,60. Diante disso, conclui-se que a implantação do projeto é economicamente viável sendo uma opção de investimento evitando o monocultivo e diversificando a produção.
\end{abstract}

PALAVRAS-CHAVE: Agricultura, economia rural, grandes culturas.

\section{ECONOMIC FEASIBILITY OF DEPLOYMENT OF SOYBEANS AND BEANS WITH CORN SUCCESSION IN SOUTHEAST GOIÁS}

\begin{abstract}
The Agriculture is one of the main economic activities of Brazil, between the main cultures, highlighting the culture of soy, beans and corn, which have a reliable supply and demand market. The present work had as objective to analyze the economic viability of the deployment of the cultivation of soya and beans with succession of corn, in the municipality of Orizona, Goiás. The property has a total area of 203.08 ha. For economic analysis have been used 130 ha, being 80 ha for cultivation of soy and 50 ha for cultivation of beans and harvest after harvest was cultivated crops with maize culture in the two areas. For the feasibility study used the following economic
\end{abstract}


indicators: index (IBC) Cost Benefit, net present value (NPV) and application of the Economic Payback of capital invested. The rate of return of $10 \%$ was used and the time of diagnosis of 5 years. It was obtained a total cost for the implementation of the project $R \$ 3.133 .188,59$, while revenue hit $R \$ 5.025 .773,97$, obtaining net profits of $\mathrm{R} \$ 1.892 .585,38$. The Payback was verified at 6 months and the reason IBC was 1.60. Given this, it is concluded that the implementation of the project is economically viable as an investment option avoiding monoculture and diversifying production.

KEYWORDS: Agriculture, rural economy, field crops.

\section{INTRODUÇÃO}

Um dos setores mais ativos da economia brasileira é constituído pelo agronegócio, destacando-se a agricultura, que exerce papel essencial para o crescimento econômico, visto que, os efeitos não se atêm somente ao mercado de produção de alimentos, mas também envolvem outros aspectos, indo desde a aquisição de insumos até a comercialização do produto final, além de oferecer matéria-prima para diversos setores (SANTOS \& VIEIRA FILHO, 2016).

Nos últimos anos o Brasil vem ganhando espaço e se concretizando como um dos principais países produtores de alimentos do mundo. No qual, especialmente nos últimos 20 anos, a produção aumentou significativamente, contribuindo para a economia. Coincide com essa cronologia a estabilização produtiva do Cerrado brasileiro, sendo a principal região produtora de grãos no país (FERNANDES \& FINCO, 2014). Os solos do cerrado apresentam alta acidez, porém com adoções de práticas de manejo como rotação de cultura, calagem, gessagem e adubação, dentre outras possibilitam o desenvolvimento de diversas culturas, garantindo assim alta produtividade. (BEZERRA et al., 2013).

Dentro deste contexto a soja é a oleaginosa de maior importância dentro do agronegócio brasileiro. Com a crescente demanda por alimentos e o aumento populacional, mantém esse produto umas das principais fontes de proteína vegetal e matéria prima para outros diversos produtos. Elemento fundamental na fabricação de rações animais e de grande importância na alimentação humana (COLUSSI et al., 2016).

Outra cultura em destaque é o feijão, presente na mesa de inúmeros brasileiros, tratado como alimentação de base. É fonte de proteína vegetal, sais minerais, vitaminas do complexo $\mathrm{B}$, ferro, cálcio e fósforo. O consumo do grão é em média, 19 quilos, por pessoa ao ano. O Brasil é o maior produtor mundial com produção média anual de 3,5 milhões de toneladas. Característico produto na nutrição brasileira é cultivado por pequenos e grandes produtores em todas as regiões (MAPA, 2016).

A cultura do milho é essencial para o setor agropecuário, a produtividade de grãos é uma atividade que tem acrescido muito nos últimos anos (NETO et al., 2016). É um dos cereais mais agricultados e produzidos no mundo, com alto valor nutricional e competência de adaptação às diversas condições ambientais, rico em carboidratos é considerado um produto energético, é utilizado na alimentação humana de forma direta ou como matéria-prima para outros produtos, é fundamental e eficiente na alimentação animal, além de ser um grande responsável pela geração de renda (SILVEIRA et al., 2015). Atualmente pode ser cultivado em todas as regiões do país e tem uma boa aceitação no cultivo da safrinha. (OLIVEIRA et al., 2014).

Para atingir o sucesso na produção são necessários os seguintes fatores: 
terra, mão de obra, capacidade empresarial, capital e tecnologia. São fatores utilizados para produzir bens ou mercadorias que recebem remunerações, tais como: aluguel, salários, lucro e royalties (COLUSSI et al., 2016). Outro fator importante que deve ser levado em consideração é a análise econômica da implantação de projetos, uma vez que, fatores como a diminuição do preço final do produto, taxas e juros altos, dificuldade de financiamentos, carência no transporte e logística, acarretam baixos retornos econômicos e até a inviabilidade do projeto, ocasionando prejuízos aos proprietários (BISOGNIN et al., 2015).

É importante salientar também que, a diversificação de culturas em uma propriedade rural pode melhorar a qualidade do solo através do processo de rotação de cultura, elevar a produtividade e diminuir os riscos econômicos do empreendimento (GLÉRIA et al., 2017). O estudo e análise dos indicadores econômicos em projetos no setor rural permitem a escolha da melhor atividade a ser implantado, com base no risco e no retorno esperado investimento, com simulações alicerçadas em parâmetros técnico-econômicos.

Em razão da escassez de informações relacionadas à viabilidade econômica na implantação de culturas agrícolas no sudeste goiano, o presente trabalho teve por objetivo analisar a viabilidade econômica na implantação do cultivo de soja e feijão com sucessão do milho em uma propriedade rural no sudeste de Goiás.

\section{MATERIAL E MÉTODOS}

O presente estudo foi realizado em uma propriedade no município de Orizona, Goiás, com as seguintes coordenadas, Longitude: 48¹7'45"O; Latitude:17 01'53"S. O clima da região de acordo com a classificação de Köppen é definido como Tropical Semi-úmido (Aw), constando temperaturas elevadas, com média anual de $22,9 \stackrel{\circ}{\circ}$ e precipitação na faixa de $1.360 \mathrm{~mm}$, com chuvas no verão e seca no inverno (ALVARES et al.,2013).

O empreendimento rural possui área total de 203,28 ha, sendo, 50,82 ha de reserva legal e área de preservação permanente, 13,46 ha de pastagem e 9 hectare para o cultivo de milho destinado a silagem para os animais. Para análise econômica foram utilizados 130 hectares, sendo 80 hectares para o cultivo da soja e 50 hectares para o cultivo do feijão na safra e após a colheita foi cultivado safrinha com a cultura do milho nas duas áreas.

No cultivo da soja (Glycine max L. Merr), para a mensuração dos cálculos foi utilizado um espaçamento de $0,5 \mathrm{~m}$ entre linhas, totalizando um estande final de 300.000 a 320.000 plantas por ha. Quanto a adubação, com base na análise de solo foi aplicado uma adubação de $200 \mathrm{~kg}$ de mono-amônio-fosfatado (MAP)/ha (11-5200) e $150 \mathrm{~kg}$ de Cloreto de potássio $(\mathrm{KCl})(00-00-60) /$ ha. Para o cultivo do feijão (Phaseolus vulgaris L.), foi utilizado um espaçamento de $0,5 \mathrm{~m}$, totalizando uma população de 150.000 plantas por ha. Quanto a adubação, também com base na análise de solo foi aplicado uma adubação de plantio com o 08-28-18 e de cobertura 20-00-20.

Ambas as áreas foram sucedidas com milho (Zea mays L.) safrinha, totalizando em 130 ha, com espaçamento de 0,5 m entre linhas, totalizando 60.000 a 70.000 plantas por ha. Na adubação no sulco de plantio foi utilizado 200 de Kg MAP e na adubação de cobertura, $200 \mathrm{Kg}$ de uréia e $120 \mathrm{~kg}$ de cloreto de potássio.

Para o diagnóstico do ambiente, foram analisados variáveis internas e externas que interferem na situação econômica. Através de vistorias in loco na propriedade, foi possível identificar os pontos fortes e fracos, as oportunidades e 
ameaças do ambiente. Também foi realizado um levantamento do patrimônio existente, dos recursos humanos, localização do empreendimento, concluindo assim a coleta de dados para o estudo de análise econômica.

De acordo com as características da propriedade, foi determinado após um estudo de mercado o que, quanto, como e para quem produzir, apurando os seguintes parâmetros: oferta e demanda, para a cultura da soja, do feijão e do milho, com visão de mercado nacional e internacional. Assim, constituindo a otimização do empreendimento, com o cultivo de soja e feijão sucedidos por milho. Como critérios de avaliação econômica utilizou-se a técnica de fluxo de caixa, Valor Presente Líquido (VPL), Índice Benefício Custo (IBC), e aplicação do Payback econômico do capital investido.

O índice Benefício/Custo: Refere-se a partir da comparação entre receitas e despesas totais atualizadas a uma taxa de juro. Sendo alcançado pela relação entre o valor da receita bruta e o valor líquido dos custos, oferecido em porcentagem (CARVALHO et al., 2016).

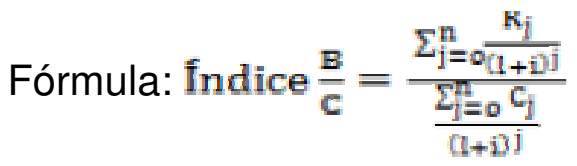

Onde:

$\mathrm{Rj}=$ receitas adquiridas no ano $\mathrm{j}$

I = taxa de desconto

$\mathrm{C} \mathrm{j}=$ custo adquiridos no ano $\mathrm{j}$

$\mathrm{n}=$ vida útil do projeto

Outro método para avaliar um investimento é o Payback atualizado. Nesta metodologia encontra o período que o investimento gasta para repor o capital investido. O payback atualizado pode ser definido como o resultado do tempo de retorno em razão do valor presente dos investimentos e valor presente dos lucros (VERGARA et al., 2017).

Fórmula: Payback atualizado $=\frac{\text { PVL (Investimentos) }}{\text { PVL (Lucros) }} \times 12$ meses ( VERGARA et al., 2017).

Enquanto que o valor presente líquido representa o valor das receitas menos o valor com o investimento inicial, quando o resultado for positivo constitui que o projeto é viável, pois o decréscimo do custo ou o retorno é maior do que o valor que foi investido (VERGARA et al., 2017).

Formula: $V L P=\sum_{j=0}^{n} R_{j}+(1+i)^{j}-\sum_{j=0}^{n} C_{j}+(1+i)^{j}$

Onde:

$\mathrm{R}_{\mathrm{j}}=$ lucros adquiridas no ano $\mathrm{j}$

$\mathrm{C}_{\mathrm{j}}=$ despesa do projeto no ano $\mathrm{j}$

$\mathrm{n}=$ relação do total de tempo do projeto

VPL $>0=0$ projeto é viável economicamente (Receita $>$ Custo);

$\mathrm{VPL}<0=0$ projeto é inviável (Receita $<$ Custo);

$\mathrm{VPL}=0=$ Custo empatado com as despesas. 
A análise de viabilidade foi feita para um período de 5 anos com uma taxa de juros de $10 \%$ ao ano (SANTOS \& GRZEBIELUCKAS, 2014).

\section{RESULTADO E DISCUSSÃO}

Para a implantação do empreendimento, os custos da cultura da soja e feijão com safrinha de milho totalizou $R \$ 684.485,31$, no qual o milho apresentou uma maior representatividade nos custos $(55,01 \%)$, em função de ser cultivado em sucessão das duas culturas (soja e feijão), sendo plantado em área total (130 ha), enquanto que a soja e o feijão foram subdivididos na área agricultável reduzindo seus custos na implantação.

A relação entre os valores das despesas e receitas por ha esta demonstrada na Tabela 1. Os valores foram estimados pelo indicador Valor presente líquido (VPL), para uma taxa de juros de $10 \%$ ao ano para um período de análise cinco de anos.

TABELA 1 - Custos e receita da implantação de soja e feijão com sucessão de milho por ha em 5 anos.

\section{Tabela de custos e receita por ha em 5 anos}

\begin{tabular}{ll}
\hline Valor total de receita & $\mathrm{R} \$ 38.659,79$ \\
Valor total de custos & $\mathrm{R} \$ 24.101,45$ \\
Valor presente líquido & $\mathrm{R} \$ 14.558 .34$
\end{tabular}

Em relação à lucratividade foi verificado o preço por saca de $60 \mathrm{~kg}$ no local de entrega dos produtos, tendo um valor pago de $R \$ 65,00$ por saca de soja, $R \$ 180,00$ por saca de feijão e $R \$ 36,50$ por saca de milho, sendo de responsabilidade do produtor a entrega dos grãos ao lugar de comercialização. Constata-se na tabela 3 os custos da produção dos 130 hectares (safra e safrinha) ao final dos 5 anos.

De acordo com os resultados, a cultura da soja apresentou $27 \%$ da receita líquida, devido ao preço de comercialização de $R \$ 65,00$. Sendo justificado por RICHETTI (2016) na safra de 2016/17, que em estudo econômico relata que quando pago valores acima de $R \$ 54,41$ por saca de $60 \mathrm{~kg}$ tem-se um retorno financeiro.

Conforme RICHETTI \& MELO (2012; 2013) a produção de feijão sequeiro, na safra de 2013/14 se demostra economicamente viável independentemente da variação de preço e quantidade. No empreendimento analisado o feijão apresentou $36 \%$ da receita líquida e foi vendido por $R \$ 180,00$ com uma produção de 44 sacas por hectare, assim caracterizando sua viabilidade.

Enquanto que a cultura de milho plantado na safrinha apresentou $37 \%$ da lucratividade do projeto, sendo a saca do grão comercializado à $R \$ 36,50$. Segundo RICHETTI et al., (2015), o milho safrinha em sistema de sequeiro demonstrou-se economicamente viável em 2016, quando os preços pagos ao produtor foram iguais e/ou superiores a $R \$ 23,07$.

$\mathrm{Na}$ tabela 3 pode-se observar os custos da produção dos 130 hectares (safra e safrinha) ao final dos 5 anos, onde através dos resultados confirma-se a viabilidade do projeto.

TABELA 3 - Custos e receitas da implantação de empreendimento em 130 ha num ENCICLOPÉDIA BIOSFERA, Centro Científico Conhecer - Goiânia, v. 14 n.25; p.1245 2017 
período de 5 anos, sendo estimados pelo indicador VPL para taxa de $10 \%$.

Tabela de custos e receita em 130 ha em de 5 anos

Valor total de custos

$\mathrm{R} \$ 3.133 .188,59$

Valor total de receita

$\mathrm{R} \$ 5.025 .773,97$

Valor presente líquido

$\mathrm{R} \$ 1.892 .585,38$

A partir dos estudos realizados, onde determina-se as análises para a implantação da cultura da soja e o feijão com segunda safra de milho, o projeto se mostra rentável para todas as culturas, como pode observar-se na tabela 4.

TABELA 4 - Indicadores de decisão econômica-financeira para a implantação do projeto.

\begin{tabular}{lc}
\hline \multicolumn{2}{c}{ Indicadores econômicos } \\
\hline VPL & $1.892 .585,38$ \\
IBC & 1,60 \\
Payback atualizado & 6 meses \\
\hline
\end{tabular}

Observa-se que o VPL é positivo (VPL>0), o IBC apresenta maior que a unidade, ou seja, que para cada $R \$ 1,00$ investido no projeto à uma taxa de desconto a $10 \%$ ao ano retorna bruto para o produtor $R \$ 1,60$ sendo o valor líquido de $R \$ 0,60$, conformando a viabilidade do empreendimento.

Nos custos de produção do projeto para as culturas da soja, feijão e milho os componentes que mais elevaram os custos foram os insumos $(81,04 \%)$, como a análise realizada por PALHETA et al., (2014), em segundo lugar têm-se custos com a colheita devido a terceirização dos maquinários ( $5 \%$ da quantidade de sacas colhidas).

$\mathrm{Na}$ análise econômica foram efetuadas simulações com o menor e o maior valor de comercialização dos produtos no ano de 2015 e o valor atual no ano de 2016, afim de contextualizar as condições reais do mercado, no qual obteve lucratividade em todos os casos.

O curto período para a recuperação do capital investido está associado a diversos fatores, tais como: a propriedade possuir maquinários e implementos agrícolas para o plantio e manutenção da lavoura, reduzindo assim o valor do investimento inicial. $E$ também pelo fato que quanto mais diversificado for a produção, maiores são as fontes de renda, assim conseguindo assegurar possíveis oscilações de mercado, o que garante a viabilidade do projeto.

No entanto, se torna necessário dissolver os custos do investimento inicial, otimizando a área, utilizando a sucessão de culturas. Tornando-se fundamental a diversificação da produção, podendo chegar a retornos suficientes para o sucesso do empreendimento (HELMERS \& SHAIK, 2003).

\section{CONCLUSÃO}

O cultivo de soja e feijão em sucessão de safrinha de milho, para as condições estabelecidas, foi considerado viável por todos os indicadores utilizados nesta análise econômica, sendo uma opção de investimento promissora para produtores do município de Orizona, Goiás. 


\section{REFERÊNCIAS}

ALVARES, C.A.; STAPE, J.L.; SENTELHAS, P.C.; GONÇALVES, J.L.M.; SPAROVEK, G. Köppen's climate classification map for Brazil. Meteorologische Zeitschrift.v.22, n. 6, p. 711-728, 2013. Disponível em: https://doi.org/10.1127/09412948/2013/0507. Doi: 10.1127/0941-2948/2013/0507

BEZERRA, R. P. M.; LOSS, A.; PEREIRA, M. G. PERIN, A. Formas de carbono em latossolosob sistemas de plantio direto e integração lavoura-pecuária no cerrado, Goiás. Semina: Ciências Agrárias, Londrina, v. 34, n. 6, p. 2637-2654, 2013. Doi: 10.5433/1679-0359.2013v34n6p2637.

BISOGNIN, M. B.; SILVA, V. R.; SOUZA, F. M.; MORO, D.; TROMBETA, H. W. Produção e comercialização agrícola em pequenas propriedades no município de Taquaruçu do Sul - RS. Enciclopédia Biosfera, Centro Científico Conhecer Goiânia, v.11 n.21. p. 2015-1542. 2015. Disponível em: http://www.conhecer.org.br/enciclop/2015b/agrarias/Producao\%20e\%20comercializa cao.pdf.

COLUSSI, J. WEISS, C. R. SOUZA, A. R. L. OLIVEIRA, L. O agronegócio da soja: Uma análise da rentabilidade do cultivo da soja no Brasil.Revista Espacios. v. 37. n.16. 2016.2 Disponível em: http://www.revistaespacios.com/a16v37n16/16371623.html.

CARVALHO, L. C.; ESPERANCINI, M. S. T.; SANTOS, J. Z.; RIBAS, L. C. Análise comparativa de estimativas de custo de produção e rentabilidade entre sojas rr1 e rr2 pro/bt ${ }^{1}$. Revista Energia na Agricultura: Botucatu, vol. 31, n.2, p.186-191, 2016. DOI: http://dx.doi.org/10.17224/EnergAgric.2016v31n2p186-191.

FERNANDES, M. da S.; FINCO, M. V. A. Sistemas de integração lavoura-pecuaria e políticas de mudanças climáticas. Pesquisa Agropecuaria Tropical. Goiânia, v.44, n.2, p.182-190, 2014.

GLÉRIA, A.A.; SILVA, R.M.; SANTOS, A.P.P.; SANTOS, KJ.G.; PAIM, T.P. Produção de bovinos de corte em sistemas de integração lavoura pecuária. Archivos de Zootecnia. Goiânia, v.66, n.66, p. 141 - 150, 2017.

HELMERS, G.; SHAIK, S. Economies of scope and scale efficiency gaints due to diversification, Western Agricultural Economics Association Annual Meeting, Denver, Colorado, p. $1-20,2003$. Disponível em: <https://core.ac.uk/download/pdf/6530936.pdf>

MAPA. MINISTÉRIO DA AGRICULTURA, PECUÁRIA E ABASTECIMENTO. Perfil do feijão no Brasil. http://www.agricultura.gov.br/vegetal/culturas/feijao/saiba-mais.

NETO, H. P. B.; GOUVEIA, J. P.; SANTOS, J. C. N.; CANUTO, D. M. F.; OLIVEIRA, R. S. Produtividade em milho safrinha sob influência da adubação nitrogenada e inoculação via foliar com azospirillum brasilense. Enciclopédia Biosfera, Centro Científico Conhecer - Goiânia, v.3, n.06. p. 45. 2016. Disponível em: 
http://www.conhecer.org.br/Agrarian\%20Academy/2016b/produtividade\%20em\%20m ilho.pdf.

OLIVEIRA, T. C.; FIGUEIREDO, Z. N.; FAVARE, H. G.; TROUI, J. G.; SILVA, R. P. Perdas quantitativas na colheita mecanizada de milho safrinha na região norte de mato grosso. Enciclopédia Biosfera, Centro Científico Conhecer - Goiânia, v.1, n.02; p. 2014. Disponível: http://www.conhecer.org.br/Agrarian\%20Academy/2014b/perdas\%20quantitativas.pd f.

PALHETA, L. C.; GOMES, C. A. S.; LOBATO, G. J. M.; PAULA, M. T.; PONTES, A. N. Viabilidade econômica de um sistema agroflorestal no município de Santa Bárbara - PA. Enciclopédia Biosfera, Centro Cientifico Conhecer - Goiânia, v.10, n.19, p.1947. 2014.

RICHETTI, A. Viabilidade Econômica da Cultura da Soja na Safra 2016/2017, em Mato Grosso do Sul. Comunicado Técnico 211, Dourados - MS, 2016. Disponível em: https://ainfo.cnptia.embrapa.br/digital/bitstream/item/146045/1/COT2016211.pdf

RICHETTI, A.; MELO, C. L. P. Viabilidade econômica da cultura do feijão-comum, safra da seca de 2014, em Mato Grosso do Sul. Comunicado Técnico 191, Dourados - $\quad$ MS, $2013 . \quad$ Disponível em: https://ainfo.cnptia.embrapa.br/digital/bitstream/item/94077/1/COT2013191.pdf

RICHETTI, A.; FLUMIGNAN, D. L.; ALMEIDA, A. C. S. Viabilidade Econômica do Milho Safrinha, Sequeiro e Irrigado, na Região Sul de Mato Grosso do Sul, para 2016. Comunicado Técnico 207, Comunicado Técnico 207, Dourados - MS, 2015. Disponível

em:

https://ainfo.cnptia.embrapa.br/digital/bitstream/item/137275/1/COT2015207.pdf

RICHETTI, A.; MELO, C. L. P. Análise da viabilidade econômica do cultivo do feijãocomum, safra 2013, em Mato Grosso do Sul. Comunicado Técnico 183, Dourados - MS, $2012 . \quad$ Disponível em: https://ainfo.cnptia.embrapa.br/digital/bitstream/item/76338/1/COT2012183.pdf

SANTOS, M. A. VIEIRA FILHO, J. E. R. 0 agronegócio brasileiro e o desenvolvimento sustentável. Ipea. Ano 13. Edição 87 - 17/06/2016. Disponível em:http://repositorio.ipea.gov.br/bitstream/11058/7122/1/O\%20agroneg\%C3\%B3cio $\% 20$ brasileiro\%20e\%200\%20desenvolvimento\%20sustent\%C3\%A1vel_Artigo_4.pdf.

SANTOS, S.S.; GRZEBIELUCKAS, C. Silvopastoral system with eucalyptus and beef cattle: na economic feasibility analysis on a farm in Mato Grosso/Brazil. Custo e Agronegócio On Line, v. 10, n. 3. p. 317-333, 2014.

SILVEIRA, D. C. BONETTI, L. P. TRAGNAGO, J. L.; NETO, N. Produtividade e características de variedades de milho crioulo cultivadas na região noroeste do Rio Grande do Sul. Enciclopédia Biosfera, Centro Científico Conhecer - Goiânia, v.2, n.04. p. 2015. Disponível em: http://www.conhecer.org.br/Agrarian\%20Academy/2015b/produtividade.pdf. 
VERGARA, W. L H.; OLIVEIRA, J. P. C.; BARBOSA, F. A.; YAMANARI, J. S. Análise de viabilidade econômico-financeira para aquisição de uma unidade de armazenagem de soja e milho. GEPROS. Gestão da Produção, Operações e Sistemas, Bauru, Ano 12, no 1, p. 41-61, 2017. DOI: 10.15675/gepros.v12i1.1598. 